\title{
Formation of Globular Clusters: In and Out of Dwarf Galaxies
}

\author{
Oleg Y. Gnedin \\ Space Telescope Science Institute, 3700 San Martin Drive, Baltimore, MD 21218, \\ USA; ognedin@stsci.edu
}

\begin{abstract}
Despite the rapid observational progress in the study of young massive star clusters, the formation of globular clusters still remains poorly understood. Yet, it is emerging that globular cluster formation is intimately linked to the formation of the Galaxy. I discuss a generic scenario of the cluster formation within progenitor galaxies, based on the available observational constraints. The oldest clusters formed around redshift $\mathrm{z}=7$, but the process continued at least until $\mathrm{z}=3$. Because of their high density, globular clusters survived when their progenitor galaxies were disrupted by the Galactic tidal field.
\end{abstract}

\section{Observational Constraints}

Building a successful physical model of globular cluster formation has proved to be an elusive topic, as revealed by the multitude of effort and the lack of result in the last 30 years. In essence, this is the point where the large-scale galaxy formation problem meets the small-scale star formation problem. In order to try to overcome the uncertainties associated with both, we should seek as many clues as possible from observations.

Fortunately, recent observational studies of star-forming regions in the Galaxy and young star clusters in interacting galaxies have improved our understanding of star formation. The majority of stars forms in clusters and associations of various sizes. The hierarchy ranges from the young OB associations to the old massive globular clusters, with no special scale between 10 and $10^{6} M_{\odot}$ : $d N / d M \propto M^{-\alpha}, \alpha=1.5-2[3,19]$. Thus globular clusters seem to represent the most massive end of the general hierarchy.

The observed luminosity function (LF) differs from the initial one due to the dynamical evolution. Small-mass clusters $\left(M<10^{5} M_{\odot}\right)$ are gradually destroyed by stellar two-body relaxation and tidal interactions [18,5, 15, 6], while very massive clusters sink to the center via dynamical friction. However, most of the high-mass clusters are essentially unaffected by the evolution, and therefore, preserve the shape of the initial LF.

Observations and modeling of old Galactic and extra-galactic globular clusters reveal similar general properties that depend little on the vastly different environment of the host galaxies (small and large, spiral and elliptical):

- Age. The oldest globular clusters are old, but realistically old: $t_{G C} \approx 12.5$ Gyr, with the $95 \%$ probability range $10.2-15.9$ Gyr 10$]$. There is no problem 
now with the stars being older than the Universe, and their age can be used to place constraints on the epoch of globular clusters formation:

$$
t_{f}=t_{H}-t_{G C},
$$

where $t_{H} \approx 13.5 \mathrm{Gyr}$ is the Hubble time for the concordance cosmological model [1]. With all the associated uncertainties, oldest clusters must have formed between redshifts $z_{f}=7$ and 3 沺.

- Metallicity. A large spread of metallicity among old clusters, from 0.01 to $0.3 Z_{\odot}$, with a median $\sim 0.03 Z_{\odot}$. The material from which globular clusters formed must have been pre-enriched by heavy elements. A narrow range of metallicity within individual clusters $(\delta[\mathrm{Fe} / \mathrm{H}]<0.1)$ indicates that each globular cluster formed in a single burst.

- Distribution. Spherically symmetric distribution and little rotation of the Galactic globular cluster system point to a different origin than most stars in the rotationally-supported disk. Also, the efficiency of globular cluster formation is universally low [13]: the ratio of the mass of globular clusters to the total baryonic (stellar + gaseous) mass of their host galaxy is the same in giant and dwarf ellipticals, $\epsilon_{G C} \equiv M_{G C} / M_{\mathrm{bar}} \approx 0.0026 \pm 0.0005$.

\section{$2 \quad$ A generic hierarchical scenario}

Early models of globular cluster formation [17,8,9] focused on local physical processes, assuming a static environment of the present Galaxy. In the last decade the paradigm of galaxy formation has changed significantly. We now have good evidence that galactic halos form as a result of gravitational growth and interaction of primordial fluctuations within the cold dark matter model 16. Small objects collapse first and merge into larger systems, extending the hierarchy to progressively higher masses. The present halo of the Milky Way formed in dozens of mergers of smaller progenitors 114,2].

At redshift $z_{f} \sim 7$, globular clusters must form within small progenitor galaxies, because large galaxies do not exist yet. The primordial gas would cool by the hydrogen recombination line emission within the first halos with the virial temperature $T_{\text {vir }}>10^{4} \mathrm{~K}$ and settle into a rotationally-supported, self-gravitating disk. Local instabilities would break the disk into dense clumps, which could further fragment into stars. Star formation in the local universe proceeds in thin disks, and it is natural to assume the same for the high-redshift progenitors. The overall spherical geometry of the globular cluster system follows from the subsequent mergers and tidal disruption of the progenitor galaxies.

For illustration, consider a simple scenario of the first episode of star formation in a progenitor galaxy. Assume that the scale-free mass function of giant molecular clouds translates into a similar cluster mass function, $d N_{c l} / d M \propto$ $M^{-\alpha}$. For $\alpha<2$, the total mass of the star cluster hierarchy within a progenitor is dominated by the most massive cluster, $M_{\max }$. Combined with the observed formation efficiency $\epsilon_{G C}$, the total baryon mass of the progenitor could be estimated 


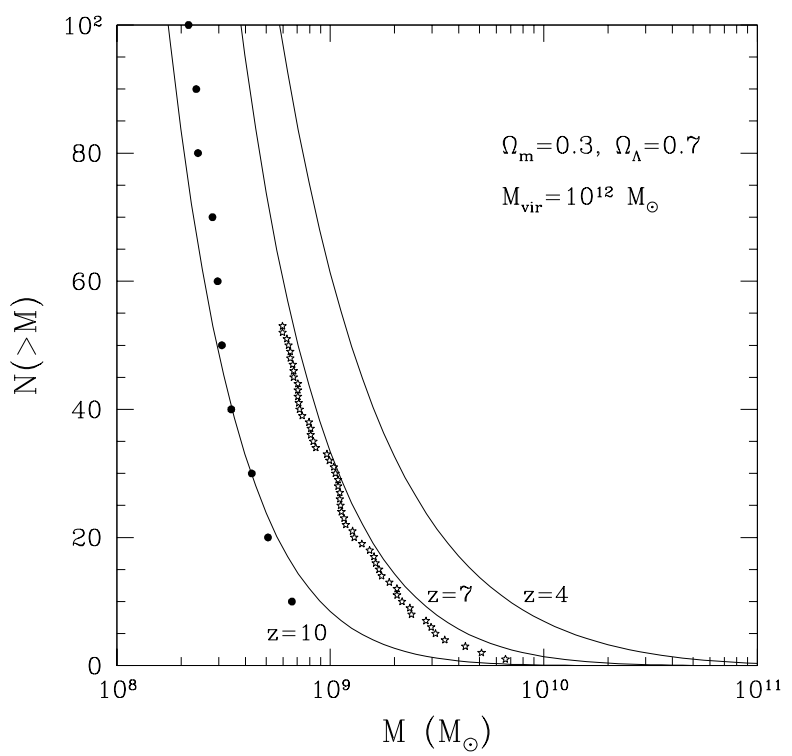

Fig. 1. Cumulative number of progenitors of the present Milky Way halo more massive than $M$ at three different redshifts. Overplotted by asterisks is the luminosity function of the massive Galactic globular clusters rescaled by a factor $\left(f_{b} \epsilon_{G C}\right)^{-1} \approx 3000$, for a constant mass-to-light ratio $M / L_{V}=2$. If some progenitors had higher formation efficiency $\epsilon_{G C}$ and others did not form globular clusters at all, the distribution would shift towards higher redshift. For example, filled circles show the mass function in the case when $10 \%$ of the progenitor halos form clusters with the efficiency $10 \epsilon_{G C}$.

as $M_{\max } / \epsilon_{G C}$. For a cosmic baryon fraction, $f_{b}=\Omega_{B} / \Omega_{0} \approx 0.13$, this corresponds to the virial mass of the progenitor $M_{\mathrm{vir}}=M_{\max } /\left(f_{b} \epsilon_{G C}\right) \approx 3000 M_{\max }$.

Figure 1 shows the distribution of the progenitor halos of the Milky Way calculated using the extended Press-Schechter formalism [12]. At redshift $z=7$, the distribution fits accurately the high end of the LF of the Galactic clusters renormalized by $\left(f_{b} \epsilon_{G C}\right)^{-1}$. In this model, the most massive cluster originates in the progenitor with the mass $M_{\text {vir }} \approx 6 \times 10^{9} M_{\odot}$ and circular velocity $v_{c} \approx 25$ $\mathrm{km} \mathrm{s}^{-1}$. Correspondingly, smaller progenitors host smaller globular clusters.

Dwarf galaxies in the Local Group provide evidence that globular clusters can form in such low potential wells. Even the Fornax dwarf spheroidal galaxy with the mass of only $2 \times 10^{7} M_{\odot}$ contains 5 clusters, while the LMC is forming star clusters now. Perhaps the most striking example is $\omega$ Cen, the most massive Galactic globular cluster. Unlike all other globular clusters, it displays multiple stellar populations with a significant spread of age $(3-5 \mathrm{Gyr})$ and metallicity $(\delta[\mathrm{Fe} / \mathrm{H}] \sim 1)$. Self-enrichment is unlikely, as this cluster is not special among others in its age and the ability to retain its own stellar winds [7]. If the oldest 

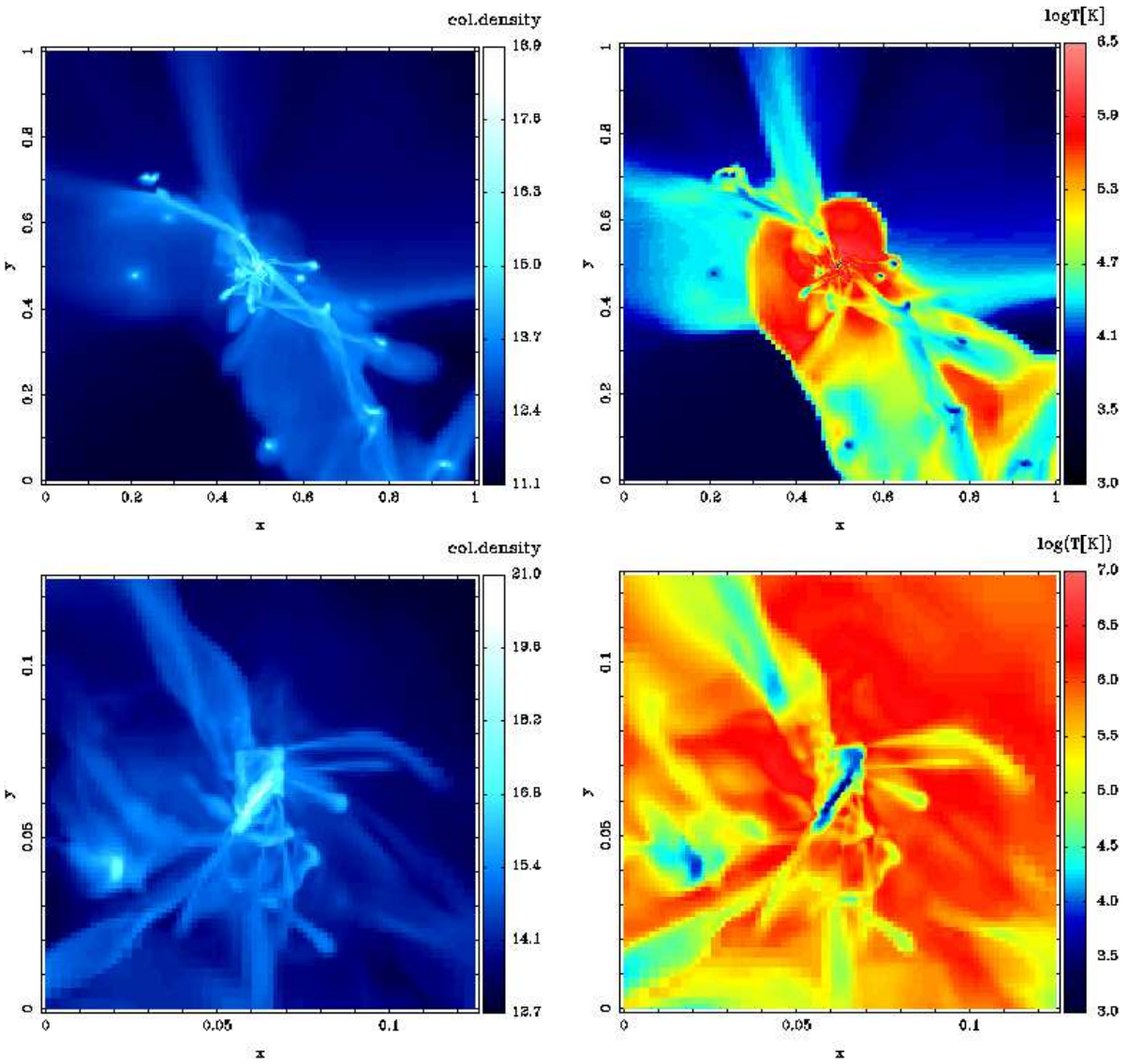

Fig. 2. ART simulation of the disk galaxy formation at $z=4$ (by A. Kravtsov). Upper panels show the gas column density and the temperature in a box of $1 h^{-1} \mathrm{Mpc}$ comoving. The lower panels show the central $125 h^{-1} \mathrm{kpc}$ around the cold disk.

stars in $\omega$ Cen had formed at its present position close to the center of the Galaxy (the pericenter is $1.2 \mathrm{kpc}$ ), the cluster would be stripped of any accumulated gas every $6 \times 10^{7} \mathrm{yr}$ and would be unable to form a second generation of stars. The only viable scenario is that it formed within a progenitor galaxy at a large distance and was brought towards the center on a very eccentric orbit.

\section{Resolving globular clusters in cosmological simulations}

The complexity of gas dynamics in the high-redshift progenitors can only be probed with direct numerical simulations. They must involve a large range of scales, from the whole galaxy to the individual clusters, and include proper tidal interactions among the progenitors. To this end, in collaboration with A. 


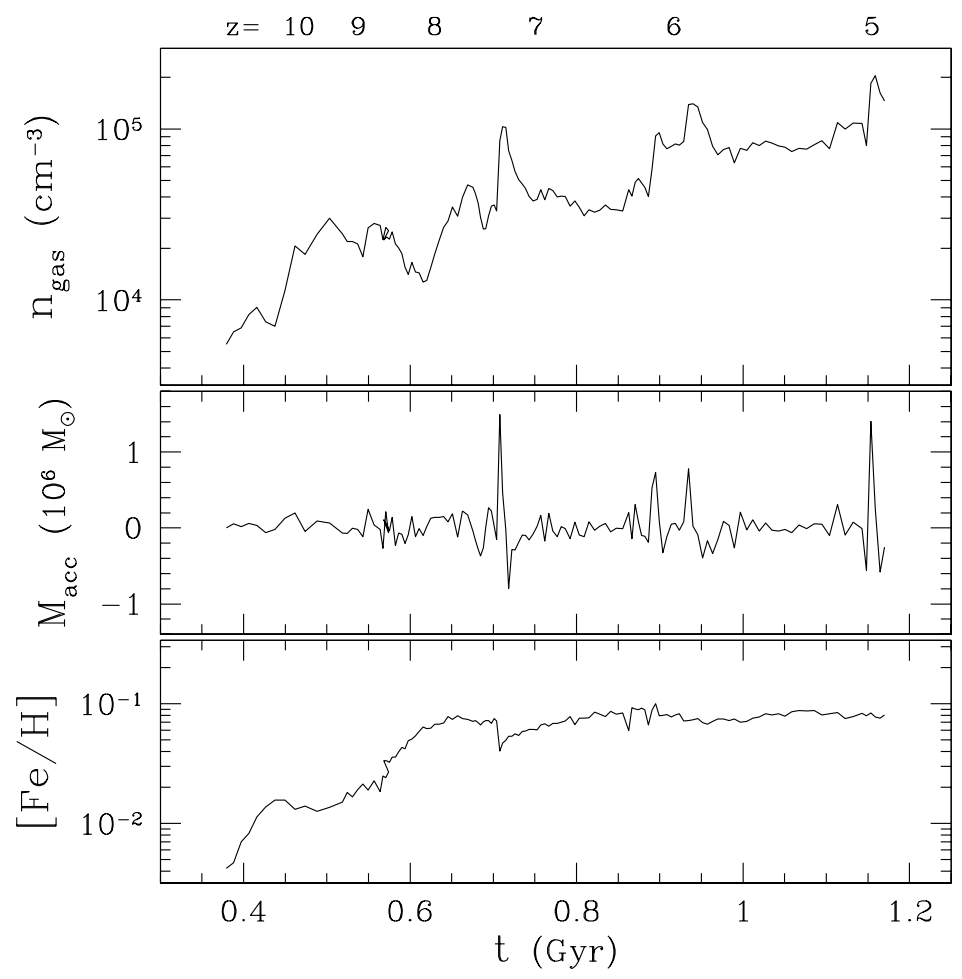

Fig. 3. ART simulation: the density, the mass accreted between successive time steps, and the metallicity of gas in the central $20 \mathrm{pc}$ of the main progenitor.

Kravtsov, we are analyzing the results of a very high-resolution simulation of the formation of a Milky Way-type galaxy. The simulation is done using the Adaptive Refinement Tree (ART) code [11], which can refine the resolution grid in the high-density regions by a factor $2^{9}$.

Figure 2 shows the distribution of gas centered on the most massive progenitor at $z=4$. The gas flows inward along the filaments and is shock-heated when it enters the dense virialized halo. A big red corona around the center in the upper right panel is the hot gas at the virial temperature. The lower panels show a thin disk which is actively forming stars in the middle; at $z=4$ its physical size is $2-4 \mathrm{kpc}$.

In the central part of the disk the gas is not allowed to cool below 300 $\mathrm{K}$, otherwise the density becomes too high and the simulation halts. This is a numerical limitation of the run which can be overcome in the future. Even with that criterion, the density in the central resolution cell exceeds $10^{5} \mathrm{~cm}^{-3}$. This is close to the density of molecular clouds and such an object is very likely to form a dense star cluster. How do we determine when a globular cluster forms?

Figure 3 shows the growth of density in the central cell, which has several noticeable peaks associated with the fast episodes of accretion. Note on the lower 
right panel of Fig. 2 that the filaments stretching all the way to the center bring in some gas that is so dense that even if shocked, it quickly cools back. Thus the central region is directly accreting cold, metal-poor gas. Such episodes of accretion or merging are apparent in the middle panel of Fig. 3, which shows the mass added between successive time steps. Some of the gas is ejected from the center by the hydrodynamical flows, so the mass difference can be negative.

The two episodes at $z \approx 7.3$ and $z \approx 5.9$ are most likely to form massive star clusters. The accreted gas suddenly increases the external pressure on the molecular clouds and triggers their collapse. The time for cluster formation is short, after 30 - 50 Myr the remaining gas may be thrown out by a new merger. After redshift of 5 the conditions become less favorable - the accretion slows down and the accumulated stellar mass starts to affect gas dynamics.

The bottom panel of Fig. 3 shows the evolution of metallicity due to the SN type II ejecta. It quickly rises to $10 \%$ of solar and then varies slowly, either up or down. Note that the sudden events of accretion of the cold, unprocessed gas lowers the mean metallicity even in the very central region. If a series of globular clusters forms between $z=8$ and 5 , the younger ones are not necessarily more metal-rich than the older ones. Thus we should not expect a clear age-metallicity correlation for the clusters formed at high redshift.

We will continue the investigation of the gas dynamics and star formation in all progenitor halos, with the goal of reproducing the present ages, metallicity, and spatial distribution of the globular clusters.

\section{References}

1. N. A. Bahcall, J. P. Ostriker, S. Perlmutter, P. J. Steinhardt: Science 284, 148 (1999)

2. J. S. Bullock, et al.: MNRAS 321, 559 (2001)

3. B. G. Elmegreen, Y. N. Efremov: ApJ 480, 235 (1997)

4. O. Y. Gnedin, O. Lahav, M. J. Rees: astro-ph/0108034 (2001)

5. O. Y. Gnedin, J. P. Ostriker: ApJ 474, 223 (1997)

6. O. Y. Gnedin, H. M. Lee, J. P. Ostriker: ApJ 522, 935 (1999)

7. O. Y. Gnedin, H.S. Zhao, J. E. Pringle, S. M. Fall, M. Livio, G. Meylan: ApJ 568, L23 (2002)

8. S. M. Fall, M. J. Rees: ApJ 298, 18 (1985)

9. W. E. Harris, R. E. Pudritz: ApJ 429, 177 (1994)

10. L. M. Krauss, B. Chaboyer: Nature, submitted; astro-ph/0111597 (2002)

11. A. V. Kravtsov, A. Klypin, Y. Hoffman: ApJ 571, 563 (2002)

12. C. Lacey, S. Cole: MNRAS 262, 627 (1993)

13. D. E. McLaughlin: AJ 117, 2398 (1999)

14. B. Moore, T. Quinn, F. Governato, J. Stadel, G. Lake: MNRAS 310, 1147 (1999)

15. C. Murali, M. D. Weinberg: MNRAS 288, 749 (1997)

16. J. P. Ostriker: Ann. Rev. Astron. Astrophys. 31, 689 (1993)

17. P. J. E. Peebles, R. H. Dicke: ApJ 154, 891 (1968)

18. L. Spitzer, Jr: Dynamical Evolution of Globular Clusters (Princeton: Princeton University Press 1987)

19. J. P. Williams, L. Blitz, C. F. McKee: in Protostars and Planets IV, p. 97 (2000) 